\title{
TWO-GENERATOR TWO-GROUPS OF CLASS TWO AND THEIR NONABELIAN TENSOR SQUARES
}

\author{
LUISE-CHARLOTTE KAPPE, MATTHEW P. VISSCHER \\ Department of Mathematical Sciences, SUNY at Binghamton, Binghamton, NY 13902-6000, USA \\ NOR HANIZA SARMIN \\ Department of Mathematics, Faculty of Science, University Technology of Malaysia, 81310 UTM, Skudai, \\ Malaysia
}

(Received 5 January, 1998)

1. Introduction. The nonabelian tensor square $G \otimes G$ of a group $G$ is generated by the symbols $g \otimes h, g, h \in G$, subject to the relations

$$
g g^{\prime} \otimes h=\left({ }^{g} g^{\prime} \otimes{ }^{g} h\right)(g \otimes h) \quad \text { and } \quad g \otimes h h^{\prime}=(g \otimes h)\left({ }^{h} g \otimes{ }^{h} h^{\prime}\right),
$$

for all $g, g^{\prime}, h, h^{\prime} \in G$, where ${ }^{g} g^{\prime}=g g^{\prime} g^{-1}$. The nonabelian tensor square is a special case of the nonabelian tensor product which has its origins in homotopy theory. It was introduced by R. Brown and J.-L. Loday in [4] and [5], extending ideas of J.H.C. Whitehead in [10]. The topic of this paper is the classification of 2-generator 2-groups of class two up to isomorphism and the determination of nonabelian tensor squares for these groups.

In [3], R. Brown, D.L. Johnson, and E.F. Robertson start the investigation of nonabelian tensor squares as group theoretical objects. One of their main goals is the explicit computation of nonabelian tensor squares. Since then many other papers have appeared on the computation of nonabelian tensor squares. For a complete overview of nonabelian tensor squares which have been determined we refer to [8]. In our context, D.L. Johnson's result on the nonabelian tensor square of a finite split metacyclic group is of interest.

THEOREM 1.1. [7] Let $G$ be a split metacyclic group given by

$$
G=\left\langle x, y \mid y^{n}=x^{m}=1, x y x^{-1}=y^{l}, l^{m} \equiv 1 \bmod n\right\rangle .
$$

Then $G \otimes G$ is the abelian group with generators $y \otimes y, x \otimes x,(x \otimes y)(y \otimes x)$, $x \otimes y$ and relations

$$
\begin{aligned}
(y \otimes y)^{n} & =(y \otimes y)^{2(l-1)}=(x \otimes x)^{m}=(x \otimes y)^{n}=1_{\otimes}, \\
((x \otimes y)(y \otimes x))^{n} & =((x \otimes y)(y \otimes x))^{l-1}=((x \otimes y)(y \otimes x))^{1+l+\ldots+l^{m-1}}=1_{\otimes}, \\
(x \otimes y)^{1+l+\ldots+l^{m-1}} & =(y \otimes y)^{(l-1)^{2} m(m-1) / 4} .
\end{aligned}
$$

To determine the nonabelian tensor squares of 2-generator 2-groups of class two we need first a classification of these groups. Trebenko in [9] attempts to classify all 2generator groups of class two. Our exposition follows along the lines of [9] and at 
the same time corrects some of the errors of that paper. In [2], also based on [9], 2generator $p$-groups of class two, $p$ an odd prime, were classified and their tensor squares determined.

As expected, the case $p=2$ is more complex since the groups in question are not regular and not necessarily split extensions. As a consequence we need to consider additional subcases when computing the nonabelian tensor squares of these groups.

In [2, Proposition 3.1] it was shown that the tensor square for a group of class two is abelian. As in [2], this fact helps us in using the concept of crossed pairing in our computations. We define it here in the case relevant for nonabelian tensor squares. For the general case of a nonabelian tensor product we refer to [3].

Definition 1.2. Let $G$ and $L$ be groups. A function $\phi: G \times G \rightarrow L$ is called a crossed pairing if

$$
\begin{aligned}
& \phi\left(g g^{\prime}, h\right)=\phi\left({ }^{g} g^{\prime},{ }^{g} h\right) \phi(g, h), \\
& \phi\left(g, h h^{\prime}\right)=\phi(g, h) \phi\left({ }^{h} g,{ }^{h} h^{\prime}\right),
\end{aligned}
$$

for all $g, g^{\prime}, h, h^{\prime} \in G$.

Crossed pairings allow us to determine homomorphic images of $G \otimes G$ as follows.

Proposition 1.3. [3] A crossed pairing $\phi: G \times G \rightarrow L$ determines a unique homomorphism of groups $\phi^{\star}: G \otimes G \rightarrow L$ such that $\phi^{\star}(g \otimes h)=\phi(g, h)$ for all $g, h \in G$.

If $G$ is a finite nilpotent group, then it follows from [3, Proposition 11] that $G \otimes G$ is the direct product of the tensor squares of the Sylow subgroups of $G$. Thus the nonabelian tensor square of a finite 2-generator group of nilpotency class two is just the direct product of the nonabelian tensor squares of $p$-groups determined here and in [2], and some abelian tensors in case of abelian Sylow components.

In conclusion, we should mention here a paper by Hartl [6]. Extending ideas from [1], he develops a method to compute nonabelian tensor squares for groups of nilpotency class two and calculates some of them for 2-generator 2-groups using this method.

2. The classification. In this section we classify all 2-generator 2-groups of nilpotency class two. First we state the familiar expansion formulae for groups of class two.

Lemma 2.1. Let $G$ be a group of nilpotency class two. Then for $x, y, z \in G$ and $n \in \mathbb{Z}$

$$
\begin{gathered}
{[x, y z]=[x, y][x, z] \quad \text { and } \quad[x y, z]=[x, z][y, z]} \\
{\left[x^{n}, y\right]=\left[x, y^{n}\right]=[x, y]^{n} \quad \text { and } \quad(x y)^{n}=x^{n} y^{n}[y, x]^{\left(\begin{array}{l}
n \\
2
\end{array}\right) .}}
\end{gathered}
$$


In the next two lemmas we state several elementary properties of 2-generator groups of nilpotency class two which are immediate corollaries of Lemma 2.1.

Lemma 2.2. Let $G=\langle a, b\rangle$ be a group of nilpotency class two. Then

(i) $G^{\prime}=\langle[a, b]\rangle$;

(ii) if $G^{\prime}$ is finite of order $m$, then $\langle a\rangle \cap Z(G)=\left\langle a^{m}\right\rangle$ and $\langle b\rangle \cap Z(G)=\left\langle b^{m}\right\rangle$.

Lemma 2.3. Let $G=\langle a, b\rangle$ be a p-group of nilpotency class two.

(i) If $G^{\prime} \cap\langle a\rangle=\left\langle a^{p^{k}}\right\rangle$ and $\left|G^{\prime}\right|=p^{\gamma}$, then $k \geq \gamma$.

(ii) If $|a|=p^{\alpha}, \alpha \geq 2,|[a, b]|=p^{\gamma}$ and $[a, b] \in\langle a\rangle$, then $\alpha \geq 2 \gamma$.

Due to the following lemma, the cases $p$ odd and $p=2$ have to be handled differently in the classification.

Lemma 2.4. Let $G$ be a nonabelian 2-generator p-group of nilpotency class two. Furthermore, let $b \in G$ be of minimal order but not in $\Phi(G)$, the Frattini subgroup of $G$, and let $a$ be an element of minimal order such that $\langle a, b\rangle=G$. If $\langle a\rangle \cap\langle b\rangle \neq\langle 1\rangle$, then $p=2$.

Proof. Choose $a$ and $b$ as in the hypothesis. Let $\langle d\rangle=\langle a\rangle \cap\langle b\rangle$. Then there exist $u, v \in \mathbb{N}$ such that $d=a^{u}=b^{v}$. Since $G$ is not cyclic, we have $u=s p^{i}, v=t p^{j}$, with $i, j \in \mathbb{N}$, and $(s, p)=(t, p)=1$. Now $|b| \leq|a|$ implies $j \leq i$. Consider $b_{1}=a^{-s p^{i-j}} b^{t}$. Since $(t, p)=1$, it follows that $\left\langle b_{1}, a\right\rangle=G$. Now by (2.1.2) and the choice of $b_{1}$, we obtain

$$
b_{1}^{p^{j}}=a^{-s p^{i}} b^{t p^{j}}\left[b^{t}, a^{-s p^{i-j}}\right]^{\left(\begin{array}{c}
p^{j} \\
2
\end{array}\right)}=[a, b]^{s t p^{i-j}\left(\begin{array}{l}
p^{j} \\
2
\end{array}\right)} .
$$

Since $b^{v}=d \neq 1$, then $|b|>p^{j}$, and by the choice of $b,\left|b_{1}\right| \geq|b|$. This implies $b_{1}^{p^{j}} \neq 1$. On the other hand, $[a, b]^{s p^{i}}=\left[a^{s p^{i}}, b\right]=\left[b^{t p^{j}}, b\right]=1$. Suppose now $p \neq 2$. For the right side of (2.4.1) we obtain $[a, b]^{s t p^{i-j}\left(\begin{array}{c}p^{j} \\ 2\end{array}\right)}=[a, b]^{s t p^{i}\left(p^{j}-1\right) / 2}=1$, a contradiction. Thus we must have $p=2$ if $\langle a\rangle \cap\langle b\rangle \neq 1$.

Now we are ready to classify 2-generator 2-groups of class two. Replacing the prime 2 by $p$, an odd prime, in (2.5.1)-(2.5.3) below and deleting $\alpha+\beta>3$ in (2.5.2) yield exactly the classification of 2-generator $p$-groups, $p$ odd, of nilpotency class two as given in (2.4.1)-(2.4.3) of [2]. We add the condition $\alpha+\beta>3$ in (2.5.2) so the dihedral group of order 8 is not included in both (2.5.1) and (2.5.2). Finally, we note that the condition $\alpha \geq \beta$ in (2.4.2) and (2.4.3) of [2] should be deleted. It appears there in error.

THEOREM 2.5. Let $G$ be a finite nonabelian 2-generator 2-group of nilpotency class two. Then $G$ is isomorphic to exactly one group of the following four types:

(2.5.1) $G \cong(\langle c\rangle \times\langle a\rangle) \rtimes\langle b\rangle$, where $[a, b]=c, \quad[a, c]=[b, c]=1, \quad|a|=2^{\alpha}$, $|b|=2^{\beta},|c|=2^{\gamma}, \alpha, \beta, \gamma \in \mathbb{N}, \alpha \geq \beta \geq \gamma$; 
(2.5.2) $G \cong\langle a\rangle \rtimes\langle b\rangle$, where $[a, b]=a^{2^{\alpha-\gamma}},|a|=2^{\alpha},|b|=2^{\beta},|[a, b]|=2^{\gamma}, \alpha, \beta$, $\gamma \in \mathbb{N}, \alpha \geq 2 \gamma, \beta \geq \gamma, \alpha+\beta>3$

(2.5.3) $G \cong(\langle c\rangle \times\langle a\rangle) \rtimes\langle b\rangle$, where $[a, b]=a^{2^{\alpha-\gamma}} c,[c, b]=a^{-2^{2(\alpha-\gamma)}} c^{-2^{\alpha-\gamma}},|a|=2^{\alpha}$, $|b|=2^{\beta},|c|=2^{\sigma},|[a, b]|=2^{\gamma}, \alpha, \beta, \gamma, \sigma \in \mathbb{N}, \beta \geq \gamma>\sigma, \alpha+\sigma \geq 2 \gamma$;

(2.5.4) $G \cong(\langle c\rangle \times\langle a\rangle)\langle b\rangle$, where $|a|=|b|=2^{\gamma+1}, \quad|[a, b]|=2^{\gamma}, \quad|c|=2^{\gamma-1}$, $[a, b]=a^{2} c,[c, b]=a^{-4} c^{-2}, a^{2^{\gamma}}=b^{2^{\gamma}}, \gamma \in \mathbb{N}$.

The groups in the above list are pairwise nonisomorphic and have nilpotency class two precisely.

Proof. Let $G$ be a finite 2-generator 2-group of nilpotency class two. Let

$$
T=\{x \in G-\Phi(G) ; x \text { has minimal order }\} .
$$

Then there are two cases:

Case I. There exist $y \in G, x \in T$ such that $G=\langle x, y\rangle$ and $\langle x\rangle \cap\langle y\rangle=1$. Among all $x \in T$ for which there is such a $y$ with $\langle x\rangle \cap\langle y\rangle=\langle 1\rangle$, choose $x=b_{1}$ such that $y=a_{1}$ is of minimal order.

Case II. For all $x \in T$ and all $y \in G$ with $\langle x, y\rangle=G,\langle x\rangle \cap\langle y\rangle \neq 1$. Among all $x \in T$ choose $x=b_{1}$ such that $y=a_{1}$ has minimal order.

From now on let $G=\left\langle a_{1}, b_{1}\right\rangle$, where $a_{1}$ and $b_{1}$ are as specified above in Case I and II, and let $\left|a_{1}\right|=2^{\alpha},\left|b_{1}\right|=2^{\beta}$ and $\left|G^{\prime}\right|=2^{\gamma}$.

Now we look at both cases separately. We first turn to Case I and consider the following five subcases:

$$
\begin{aligned}
& \left\langle a_{1}\right\rangle \cap G^{\prime}=\langle 1\rangle, \quad\left\langle b_{1}\right\rangle \cap G^{\prime}=\langle 1\rangle ; \\
& \left\langle b_{1}\right\rangle \cap G^{\prime}=\langle 1\rangle, \quad\left\langle a_{1}\right\rangle \cap G^{\prime}=G^{\prime} ; \\
& \left\langle b_{1}\right\rangle \cap G^{\prime}=\langle 1\rangle, \quad\langle 1\rangle \neq\left\langle a_{1}\right\rangle \cap G^{\prime} \subset G^{\prime} ; \\
& \left\langle a_{1}\right\rangle \cap G^{\prime}=\langle 1\rangle, \quad\left\langle b_{1}\right\rangle \cap G^{\prime}=G^{\prime} ; \\
& \left\langle a_{1}\right\rangle \cap G^{\prime}=\langle 1\rangle, \quad\langle 1\rangle \neq\left\langle b_{1}\right\rangle \cap G^{\prime} \subset G^{\prime} .
\end{aligned}
$$

Suppose (2.5.5) holds. Set $b=b_{1}$ and $a=a_{1}$. Now $\alpha \geq \beta$ follows by choice of $a$ and $b$, and $|b|=2^{\beta}$ implies $1=\left[a, b^{2^{\beta}}\right]=[a, b]^{2^{\beta}}$. Thus $\beta \geq \gamma$. This together with $G^{\prime} \neq\langle 1\rangle$ yields $\alpha \geq \beta \geq \gamma \geq 1$. Then it is easy to see that $G=(\langle[a, b]\rangle \times\langle a\rangle) \rtimes\langle b\rangle$, and $G$ is of type (2.5.1).

Next consider (2.5.6). We have $\left[a_{1}, b_{1}\right]=a_{1}^{r^{2-\gamma}}$ for some odd integer $r$. Setting $a=a_{1}$ and $b=b_{1}^{s}$ with $r s \equiv 1\left(\bmod 2^{\gamma}\right)$, we obtain $[a, b]=a_{1}^{r s 2^{\alpha-\gamma}}=a^{2^{\alpha-\gamma}}$. It is easy to see that $G$ is of type (2.5.2).

Now suppose (2.5.7) holds. Set $c_{1}=\left[a_{1}, b_{1}\right]$. We have $\left\langle c_{1}^{2^{\sigma}}\right\rangle=\left\langle a_{1}\right\rangle \cap G^{\prime}$ for some $\sigma \in \mathbb{N}$, and $\sigma$ is minimal such that $1 \neq c_{1}^{2^{\sigma}}=a_{1}^{s k^{k}}$ for $s, k \in \mathbb{N}$ and $(s, 2)=1$. It follows $\sigma<\gamma$. Furthermore, $2^{\gamma-\sigma}=\left|c_{1}^{2^{\sigma}}\right|=\left|a_{1}^{s 2^{k}}\right|=2^{\alpha-k}$. This implies $k=\alpha-\gamma+\sigma$. By (i) of Lemma 2.3 we obtain $\alpha+\sigma \geq 2 \gamma$. Set $a=a_{1}^{s}$ and $c=c_{1} a^{-2^{k-\sigma}}=c_{1} a^{-2^{\alpha-\gamma}}$, thus $c^{2^{\sigma}}=1$. This together with the minimality of $\sigma$ implies $|c|=2^{\sigma}$ and $\left|G^{\prime} \cap\langle a\rangle\right|=2^{\gamma-\sigma}$. It can be shown that $\langle c\rangle \cap\langle a\rangle=\langle 1\rangle$, and thus $\left\langle c_{1}, a\right\rangle=\langle c, a\rangle=\langle c\rangle \times\langle a\rangle$. 
Set $b_{1}=b$. We claim $\langle c\rangle \cap\langle b\rangle=\langle 1\rangle$. Suppose the contrary. Since $c \in\langle[a, b]$, $\left.a^{-2^{k-\sigma}}\right\rangle$, this would mean that there exist integers $\lambda, \mu \geq 0$ such that $a^{-2^{k-\sigma+\lambda}} \equiv b^{r 2^{\mu}}$ $\bmod G^{\prime}$ for some $r \in \mathbb{N}$ with $(r, 2)=1$. Suppose $a^{-2^{k-\sigma+\lambda}} \not \equiv 1 \bmod G^{\prime}$. Setting $i=\alpha-k+\sigma-\lambda-1$, we have $a^{-2^{k-\sigma+\lambda+i}}=a^{-2^{\alpha-1}}$. Thus $a^{-2^{k-\sigma+\lambda+i}}$ has order 2. Since $k \leq \alpha-1, a^{-2^{\alpha-1}} \in G^{\prime}$. Therefore $1 \neq b^{2^{\mu+i}} \in\langle a\rangle \cap G^{\prime}$, and hence $\langle a\rangle \cap\langle b\rangle \neq\langle 1\rangle$, a contradiction to the choice of $a$ and $b$. If already $a^{-2^{k-\sigma+\lambda}} \equiv 1 \bmod G^{\prime}$, we arrive at the same contradiction. Thus we conclude that $\langle c\rangle \cap\langle b\rangle=\langle 1\rangle$. It can be shown that $G$ is of type (2.5.3).

The cases (2.5.8) and (2.5.9) are similar to (2.5.6) and (2.5.7) where the roles of $a$ and $b$ are just interchanged. Specifically, suppose (2.5.8) or (2.5.9) hold and $G=\left\langle a_{1}, b_{1}\right\rangle$. Here we set $a=b_{1}$ and $b=a_{1}^{s}$ for a suitable odd integer $s$ and arrive at a group of type (2.5.2) or (2.5.3), respectively.

Finally, we consider Case II, i.e for all $x \in T$ and all $y \in G$ with $G=\langle x, y\rangle$ we have $\langle x\rangle \cap\langle y\rangle \neq\langle 1\rangle$. There exist odd integers $s, t$, such that $a_{1}^{s 2^{k}}=b_{1}^{t 2^{l}}$, for $k, l \in \mathbb{N}$. Since $\left|b_{1}\right| \leq\left|a_{1}\right|$, we have $l \leq k$. We claim that $k=\gamma$. Let $b_{0}=a_{1}^{-s 2^{k-l}} b_{1}^{t}$. Since $(t, 2)=1$, it follows that $\left\langle b_{0}, a_{1}\right\rangle=G$, and (2.4.1) yields

$$
b_{0}^{2^{l}}=\left[a_{1}, b_{1}\right]^{s t 2^{k-1}\left(2^{l}-1\right)} .
$$

By the choice of $b_{1}$ and $\left|b_{0}\right| \geq\left|b_{1}\right|>2^{l}$, we obtain $b_{0}^{2^{l}} \neq 1$. Thus $\left[a_{1}, b_{1}\right]^{2^{k-1}} \neq 1$. Since $s$ and $t$ are odd, $\left[a_{1}, b_{1}\right]^{s 2^{k}}=\left[a_{1}^{s 2^{k}}, b_{1}\right]=\left[b_{1}^{t 2^{l}}, b_{1}\right]=1$. This implies $k=\gamma$.

We will show $l=\gamma$ as well. Note that by (2.1.2) we obtain $\left[a_{1}, b_{1}\right]^{t 2^{l}}=\left[a_{1}, b_{1}^{t 2^{l}}\right]=\left[a_{1}, a_{1}^{s 2^{\gamma}}\right]=1$. Hence $l \geq \gamma$. However, by the above and our choice of $b_{1}$, we have $l \leq k=\gamma$, and the conclusion follows.

Now we claim that $\left|b_{0}\right|=\left|b_{1}\right|=\left|a_{1}\right|=2^{\gamma+1}$. $>$ From (2.5.10) and the above it follows $b_{0}^{2^{\gamma}} \neq 1$ and $b_{0}^{2^{\gamma+1}}=\left[a_{1}, b_{1}\right]^{s 2^{\gamma}\left(2^{\gamma}-1\right)}=1$. Hence $\left|b_{0}\right|=2^{\gamma+1}$. This together with $\left|b_{0}\right| \geq\left|b_{1}\right|>2^{\gamma}$ yields $\left|b_{1}\right|=2^{\gamma+1}$. Since $k=l$, we have $a_{1}^{-s}=b_{0} b_{1}^{-t}$, and therefore by (2.1.2), $\left(a_{1}^{-s}\right)^{2^{\gamma+1}}=\left[a_{1}, b_{1}\right]^{s t\left(\begin{array}{c}2^{\gamma+1} \\ 2\end{array}\right)}=1$. This implies $\left|a_{1}\right| \leq 2^{\gamma+1}$. But by the above $\left|a_{1}\right|>2^{\gamma}$. We conclude $\left|a_{1}\right|=2^{\gamma+1}$.

Since $\left|b_{0}\right|=\left|b_{1}\right|$ and $\left\langle b_{0}, a_{1}\right\rangle=\left\langle b_{1}, a_{1}\right\rangle$, we have $b_{0} \in T$. We set $b=b_{0}$ and $a=a_{1}$, and thus obtain $a^{2^{\gamma}}=b^{2^{\gamma}}$. By (ii) of Lemma 2.2, we have $\left\langle b^{2^{\gamma}}\right\rangle=\langle b\rangle \cap Z(G) \supseteq G^{\prime} \cap\langle b\rangle \neq 1$. Since $\left|b^{2^{\gamma}}\right|=2$, this and the above yield $\langle b\rangle \cap G^{\prime}=\langle a\rangle \cap G^{\prime}=\left\langle b^{2^{\gamma}}\right\rangle$. It can be easily verified that $Z(G)=G^{\prime}$.

First let $\gamma=1$. Then $|a|=|b|=2^{2}, a^{2}=b^{2}=[a, b]$, and $\mathrm{G}$ is the group of quaternions of order 8 . For $\gamma>1$, set $c=a^{-2}[a, b]$. Since $[a, b]^{2^{\gamma-1}}=a^{2^{\gamma}}$, we obtain $c^{2^{\gamma-1}}=\left(a^{-2}[a, b]\right)^{2^{\gamma-1}}=a^{2^{\gamma}}[a, b]^{2^{\gamma-1}}=1$. This together with $\langle a\rangle \cap G^{\prime}=\left\langle a^{2^{\gamma}}\right\rangle$ implies $|c|=2^{\gamma-1}$. We claim $\langle a\rangle \cap\langle c\rangle=\langle 1\rangle$. Let $\langle d\rangle=\langle a\rangle \cap\langle c\rangle$. Then there exist integers $n, m$ such that $a^{n}=c^{m}=d$, and therefore $a^{n+2 m}=[a, b]^{m}$. Since $G^{\prime} \cap\langle a\rangle=\left\langle a^{2^{\gamma}}\right\rangle$, this yields $m \equiv 2^{\gamma-1}\left(\bmod 2^{\gamma}\right)$. Since $|c|=2^{\gamma-1}$, we obtain $1=c^{m}=d$. Thus $\langle a\rangle \cap\langle c\rangle=\langle 1\rangle$ and $\langle a, c\rangle=\langle a\rangle \times\langle c\rangle$. To construct the group $G$, consider $H=(\langle w\rangle \times\langle u\rangle) \rtimes\langle v\rangle$, a group of type (2.5.3) with $\alpha=\beta=\gamma+1$. Since $u^{2^{\gamma}}, v^{2^{\gamma}} \in Z(H),\left\langle u^{2^{\gamma}} v^{2^{\gamma}}\right\rangle \triangleleft H$. Consider $G=H /\left\langle u^{2^{\gamma}} v^{2^{\gamma}}\right\rangle$. It can be shown that $G$ is of type (2.5.4).

It is easy to verify that all groups in the above list have nilpotency class two. It is straightforward but lengthy to show that they are all pairwise nonisomorphic.

3. The tensor square of groups of class 2 . In this section we include some general results about nonabelian tensor squares, from here on referred to as tensor squares, 
as well as a few results specific to groups of nilpotency class two. We also include a number theoretic lemma. These results will be used in the next section.

Proposition 3.1. Let $G, H, K, L$ be groups with $\pi: H \rightarrow G$ an epimorphism, $\varphi: K \rightarrow L$ a homomorphism, and $\Gamma: H \times H \rightarrow K$ a crossed pairing. If $\Gamma(\operatorname{ker} \pi, H)$ and $\Gamma(H, \operatorname{ker} \pi)$ are contained in $\operatorname{ker} \varphi$, then there exists a crossed pairing $\Delta: G \times G \rightarrow L$ for which the following diagram commutes:

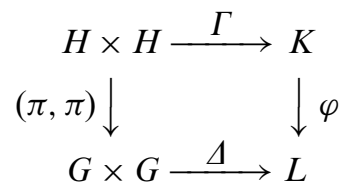

Proof. Since $\pi$ is an epimorphism we have $i: G \rightarrow H / \operatorname{ker} \pi$ is an isomorphism. We define a mapping $\Delta^{\prime}: H / \operatorname{ker} \pi \times H / \operatorname{ker} \pi \rightarrow L$ by $\Delta^{\prime}\left(h \operatorname{ker} \pi, h^{\prime} \operatorname{ker} \pi\right)=$ $\varphi\left(\Gamma\left(h, h^{\prime}\right)\right)$, where $h, h^{\prime} \in H$. We claim that $\Delta^{\prime}$ is a crossed pairing. First we show $\Delta^{\prime}$ is well-defined. Note that for $h_{1}, h_{2}, h^{\prime} \in H$ we have $\Gamma\left(h_{2} h_{1}^{-1}, h^{\prime}\right)=\Gamma\left(h_{1}^{-1}\left({ }^{h_{1}} h_{2}\right), h^{\prime}\right)$, and hence

$$
\Gamma\left(h_{2} h_{1}^{-1}, h^{\prime}\right)=\Gamma\left(h_{2}, h^{\prime}\right) \cdot \Gamma\left(h_{1}, h^{\prime}\right)^{-1} .
$$

Now suppose $h_{1}, h_{2} \in H$ so that $h_{1} \operatorname{ker} \pi=h_{2} \operatorname{ker} \pi$. Then, since $\Gamma(\operatorname{ker} \pi, H) \subseteq \operatorname{ker} \varphi$ and $h_{2} h_{1}^{-1} \in \operatorname{ker} \pi$, (3.1.1) yields $1_{L}=\varphi\left(\Gamma\left(h_{2} h_{1}^{-1}, h^{\prime}\right)\right)=\varphi\left(\Gamma\left(h_{2}, h^{\prime}\right)\right) \cdot \varphi\left(\Gamma\left(h_{1}, h^{\prime}\right)\right)^{-1}$. Therefore $\varphi\left(\Gamma\left(h_{1}, h^{\prime}\right)\right)=\varphi\left(\Gamma\left(h_{2}, h^{\prime}\right)\right)$. Similarly, it follows $\varphi\left(\Gamma\left(h^{\prime}, h_{1}\right)\right)=\varphi\left(\Gamma\left(h^{\prime}, h_{2}\right)\right)$. We conclude that $\Delta^{\prime}$ is well-defined. It can be easily verified that $\Delta^{\prime}$ is a crossed pairing.

For $\Delta=\Delta^{\prime} \circ(i, i)$ the diagram commutes. This can be seen as follows. Let $h, h^{\prime} \in H$. Then $\Delta \circ(\pi, \pi)\left(h, h^{\prime}\right)=\Delta\left(\pi(h), \pi\left(h^{\prime}\right)\right)=\varphi\left(\Gamma\left(h, h^{\prime}\right)\right)$. So $\Delta \circ(\pi, \pi)=\varphi \circ \Gamma$.

The following 3 lemmas summarize results about tensor squares of nilpotent groups of class two. They, together with their proofs, can be found in [2].

Lemma 3.2. [2] Let $G$ be a group of nilpotency class two; then for any $x, y \in G$ and $m, n \in \mathbb{Z}$,

$$
x^{n} \otimes y^{m}=(x \otimes y)^{n m}(y \otimes[x, y])^{n\left(\begin{array}{c}
m \\
2
\end{array}\right)}(x \otimes[x, y])^{m\left(\begin{array}{c}
n \\
2
\end{array}\right)} .
$$

For a 2-generator group of class two we have the following expansion formula.

Lemma 3.3. [2] Let $G=\langle a, b\rangle$ be a group of class two, and $g, h \in G$ with $g=a^{m} b^{n} z^{l}$ and $h=a^{m^{\prime}} b^{n^{\prime}} z^{l^{\prime}}$, where $m, m^{\prime}, n, n^{\prime}, l, l^{\prime} \in \mathbb{Z}$, and $z=[a, b]$. Then

$$
g \otimes h=(a \otimes a)^{\alpha_{1}}(b \otimes b)^{\alpha_{2}}(a \otimes b)^{\alpha_{3}}(b \otimes a)^{\alpha_{4}}(a \otimes z)^{\alpha_{5}}(b \otimes z)^{\alpha_{6}},
$$

where

$$
\begin{aligned}
& \alpha_{1}=m m^{\prime}, \alpha_{2}=n n^{\prime}, \alpha_{3}=m n^{\prime}, \alpha_{4}=n m^{\prime}, \\
& \alpha_{5}=m l^{\prime}-m^{\prime} l+n^{\prime}\left(\begin{array}{c}
m \\
2
\end{array}\right)-n\left(\begin{array}{c}
m^{\prime} \\
2
\end{array}\right)+m m^{\prime}\left(n^{\prime}-n\right), \\
& \alpha_{6}=n l^{\prime}-n^{\prime} l+m\left(\begin{array}{l}
n^{\prime} \\
2
\end{array}\right)-m^{\prime}\left(\begin{array}{l}
n \\
2
\end{array}\right) .
\end{aligned}
$$


In case of the Heisenberg group $\mathcal{H}$, the free group of class two and rank two, the above expansion leads to a crossed pairing.

Lemma 3.4. [2] Let $\mathcal{H}=F_{2} / \gamma_{3}\left(F_{2}\right)=\langle x, y\rangle$ and $g, h \in \mathcal{H}$ with $g=x^{m} y^{n} z^{l}$ and $h=x^{m^{\prime}} y^{n^{\prime}} z^{l^{\prime}}$, where $m, m^{\prime}, n, n^{\prime}, l, l^{\prime} \in \mathbb{Z}$, and $z=[x, y]$. Then the mapping $\psi: \mathcal{H} \times \mathcal{H} \rightarrow \mathbb{Z}^{6}$, defined componentwise as $\psi(g, h)=\left(x_{1}(g, h), \ldots, x_{6}(g, h)\right)$, where

$$
\begin{aligned}
& x_{1}(g, h)=m m^{\prime}, \quad x_{2}(g, h)=n n^{\prime}, \quad x_{3}(g, h)=m n^{\prime}, \quad x_{4}(g, h)=n m^{\prime}, \\
& x_{5}(g, h)=m l^{\prime}-m^{\prime} l+n^{\prime}\left(\begin{array}{c}
m \\
2
\end{array}\right)-n\left(\begin{array}{c}
m^{\prime} \\
2
\end{array}\right)+m m^{\prime}\left(n^{\prime}-n\right), \\
& x_{6}(g, h)=n l^{\prime}-n^{\prime} l+m\left(\begin{array}{c}
n^{\prime} \\
2
\end{array}\right)-m^{\prime}\left(\begin{array}{l}
n \\
2
\end{array}\right),
\end{aligned}
$$

is a crossed pairing.

Finally, we will need the following number theoretic lemma in the next section.

Lemma 3.5. Let $\alpha, \beta, \gamma \in \mathbb{N}$ such that $2 \gamma \leq \alpha, \gamma \leq \beta$. For $n \in \mathbb{N}$ denote by $[n]_{2}$ the highest power of 2 dividing $n$. Then

$$
\begin{gathered}
\left(2^{\alpha}-2^{\alpha-\gamma}+1\right)^{2^{\beta}} \equiv 1\left(\bmod 2^{\alpha}\right) \\
{\left[\sum_{k=0}^{2^{\beta}-1}\left(2^{\alpha}-2^{\alpha-\gamma}+1\right)^{k}\right]_{2}= \begin{cases}\beta+1, & \text { if } \alpha=2 \text { and } \gamma=1, \\
\beta, & \text { otherwise. }\end{cases} }
\end{gathered}
$$

The verification of (3.5.1) and (3.5.2) is straightforward and will be omitted here.

4. Computation of the tensor squares. In this section we determine the tensor squares of the groups classified in Section 2. According to Theorem 2.5, we have to deal with four different cases. We start with groups of type (2.5.2). Such groups are split cyclic extensions, and thus we can use Theorem 1.1 to compute the tensor square.

THEOREM 4.1. Let $G$ be a group of type (2.5.2), that is $G \cong\langle a\rangle \rtimes\langle b\rangle$, where $[a, b]=a^{2^{\alpha-\gamma}}, \quad|a|=2^{\alpha}, \quad|b|=2^{\beta}, \quad|[a, b]|=2^{\gamma}, \quad \alpha, \beta, \gamma \in \mathbb{N}$ with $\alpha \geq 2 \gamma, \quad \beta \geq \gamma$, $\alpha+\beta>3$. Then

$$
G \otimes G \cong \mathbb{Z}_{2^{\alpha-\gamma+1}} \times \mathbb{Z}_{2^{\beta}} \times \mathbb{Z}_{2^{\min \{\alpha-\gamma, \beta\}}} \times \mathbb{Z}_{2^{\min \{\alpha, \beta\}}} .
$$

Proof. Let $G$ be a group of type (2.5.2). Obviously, $G$ is a metacylic split extension generated by $a, b$, subject to the defining relations $a^{2^{\alpha}}=b^{2^{\beta}}=1$, and $b a b^{-1}=a^{\alpha^{\alpha}-2^{\alpha-\gamma}+1}$. By (3.5.1) we have $\left(2^{\alpha}-2^{\alpha-\gamma}+1\right)^{2^{\beta}} \equiv 1\left(\bmod 2^{\alpha}\right)$. Thus $G$ satisfies the conditions of Theorem 1.1 with $x=b, y=a, m=2^{\beta}, \quad n=2^{\alpha}$ and 
$l=2^{\alpha}-2^{\alpha-\gamma}+1$. Therefore $G \otimes G$ is the abelian group generated by $a \otimes a, b \otimes b$, $(b \otimes a)(a \otimes b)$, and $b \otimes a$ with relations as given in Theorem 1.1; in particular $\langle b \otimes a\rangle \cap\langle a \otimes a\rangle=\left\langle(a \otimes a)^{k}\right\rangle$, where $k=\frac{1}{4}(l-1)^{2} m(m-1)$. We will show that $(a \otimes a)^{k}=1_{\otimes}$ under our assumption that $\alpha+\beta>3$. By Theorem 1.1 we have $(a \otimes a)^{2(l-1)}=1_{\otimes}$. To prove our claim it suffices to show that $(l-1) m \equiv 0\left(\bmod 2^{3}\right)$. We observe $[(l-1) m]_{2}=\alpha+\beta-\gamma$. By (2.5.2) we have $\alpha \geq 2 \gamma$ and $\beta \geq \gamma$. If $\alpha>2 \gamma$ or $\beta>\gamma$, then clearly $\alpha+\beta-\gamma \geq 3$. If $\alpha=2 \gamma$ and $\beta=\gamma$, then $\alpha+\beta-\gamma=2 \gamma$. Since $\alpha+\beta>3$, this implies $\alpha+\beta-\gamma>3$. Thus $2(l-1)$ divides $k$ and $(a \otimes a)^{k}=1_{\otimes}$. By Theorem 1.1 we have now $G \otimes G=\langle a \otimes a\rangle \times\langle b \otimes b\rangle \times$ $\langle(b \otimes a)(a \otimes b)\rangle \times\langle b \otimes a\rangle$.

All that remains to be done is to calculate the orders of the factors explicitly. The orders of the first and the second factors are $(n, 2(l-1))=2^{\alpha-\gamma+1}$ and $2^{\beta}$, respectively. The order of the third factor is $\left(n, l-1,1+l+\ldots+l^{m-1}\right)$, while the order of the fourth factor is $\left(n, 1+l+\ldots+l^{m-1}\right)$. First suppose $\alpha=2$ and $\gamma=1$. Then by (3.5.2) we obtain $\left(n, l-1,1+l+\ldots+l^{m-1}\right)=\left(2^{2}, 2,2^{\beta+1}\right)=2$ and $\left(n, 1+l+\ldots+l^{m-1}\right)=\left(2^{2}, 2^{\beta+1}\right)=2^{2}$. We conclude $G \otimes G \cong \mathbb{Z}_{2^{2}}^{2} \times \mathbb{Z}_{2^{\beta}} \times \mathbb{Z}_{2}$ in this case. But $\alpha=2$ and $\gamma=1$ implies $2=\alpha-\gamma+1,1=\min \{\alpha-\gamma, \beta\}$, and $2=\min \{\alpha, \beta\}$. So we get the desired result. Now suppose otherwise. By (3.5.2), we have $\left(n, l-1,1+l+\ldots+l^{m-1}\right)=\left(2^{\alpha}, 2^{\alpha}-2^{\alpha-\gamma}, 2^{\beta}\right)=\min \left\{2^{\alpha-\gamma}, 2^{\beta}\right\} \quad$ and $\left(n, 1+l+\ldots+l^{m-1}\right)=\left(2^{\alpha}, 2^{\beta}\right)=\min \left\{2^{\alpha}, 2^{\beta}\right\}$, the desired result.

Next we compute the nonabelian tensor square of a group of type (2.5.1). The proof follows along the lines of [2, Theorem 4.3], addressing the corresponding case for $p \neq 2$. However, we have to deal with one exception for $p=2$.

THEOREM 4.2. Let $G$ be a group of type (2.5.1), that is $G \cong(\langle c\rangle \times\langle a\rangle) \rtimes\langle b\rangle$, where $[a, b]=c,[a, c]=[b, c]=1,|a|=2^{\alpha},|b|=2^{\beta},|c|=2^{\gamma}, \alpha, \beta, \gamma \in \mathbb{N}, \alpha \geq \beta \geq \gamma$. Then

$$
G \otimes G \cong \begin{cases}\mathbb{Z}_{2^{\alpha}} \times \mathbb{Z}_{2^{\beta}}^{3} \times \mathbb{Z}_{2^{\gamma}}^{2}, & \text { if } \beta>\gamma, \\ \mathbb{Z}_{2^{\alpha}} \times \mathbb{Z}_{2^{\gamma}} \times \mathbb{Z}_{2^{\gamma+1}} \times \mathbb{Z}_{2^{\gamma}} \times \mathbb{Z}_{2^{\min \{\alpha-1, \gamma\}} \times \mathbb{Z}_{2^{\gamma-1}},} & \text { if } \beta=\gamma .\end{cases}
$$

Proof. Let $G$ be a group of type (2.5.1). We have two cases, namely $\beta>\gamma$ and $\beta=\gamma$. The first case follows from [2, Theorem 4.3], setting $p=2$ and $\beta>\gamma$. So we omit the details.

Now let $\beta=\gamma$. From Lemma 3.3 it follows that

$G \otimes G=\left\langle a \otimes a, b \otimes b, a \otimes b,(a \otimes b)(b \otimes a),(a \otimes b)^{2}(a \otimes c),(a \otimes b)^{2}(b \otimes c)\right\rangle$.

With this generating set we show $G \otimes G$ has at most the order in question. Direct expansion yields $(a \otimes a)^{2^{\alpha}}=(b \otimes b)^{2^{\gamma}}=1_{\otimes}$. For the next two generators, expansion using Lemma 3.2 yields $1_{\otimes}=\left(a \otimes b^{2^{\gamma}}\right)^{2}=\left((a \otimes b)^{2^{\gamma}}(b \otimes c)^{2^{\gamma-1}}\right)^{2}=(a \otimes b)^{2^{\gamma+1}}$. $(b \otimes c)^{2^{\gamma}}=(a \otimes b)^{2^{\gamma+1}}$, and $1_{\otimes}=\left(a \otimes b^{2^{\gamma}}\right)\left(b^{2^{\gamma}} \otimes a\right)=(a \otimes b)^{2^{\gamma}}(b \otimes c)^{2^{\gamma-1}}(b \otimes a)^{2^{\gamma}}$. $(b \otimes c)^{-2^{\gamma-1}}=((a \otimes b)(b \otimes a))^{2^{\gamma}}$. Next consider $(a \otimes b)^{2}(a \otimes c)$. By Lemma 3.2, $1_{\otimes}=a^{2^{\alpha}} \otimes b=(a \otimes b)^{2^{\alpha}}(a \otimes c)^{2^{\alpha-1}}=\left((a \otimes b)^{2}(a \otimes c)\right)^{2^{\alpha-1}}, \quad$ and $\quad 1_{\otimes}=\left(a \otimes b^{2^{\gamma+1}}\right)$. $\left(a \otimes c^{2^{\gamma}}\right)=(a \otimes b)^{2^{\gamma+1}}(a \otimes c)^{2^{\gamma}}=\left((a \otimes b)^{2}(a \otimes c)\right)^{2^{\gamma}}$. Hence $\quad\left|(a \otimes b)^{2}(a \otimes c)\right|$ $\leq 2^{\min \{\alpha-1, \gamma\}}$. Finally, in a similar fashion using Lemma 3.2, $1_{\otimes}=a \otimes b^{2^{\gamma}}=$ $\left((a \otimes b)^{2}(b \otimes c)\right)^{2^{\gamma-1}}$. Thus $|G \otimes G| \leq 2^{\alpha+4 \gamma+\min \{\alpha-1, \gamma\}}$. 
Let $\mathcal{H}=F_{2} / \gamma_{3}\left(F_{2}\right)=\langle x, y\rangle$. Define $\pi: \mathcal{H} \rightarrow G$ by $\pi(h)=a^{m} b^{n} c^{l}$ for $h \in \mathcal{H}$, where $h=x^{m} y^{n} z^{l}, m, n, l \in \mathbb{Z}, z=[x, y]$. It follows that $\pi$ is a homomorphism onto $G$. Next set $L=\mathbb{Z}_{2^{\alpha}} \times \mathbb{Z}_{2^{\gamma}} \times \mathbb{Z}_{2^{\gamma+1}} \times \mathbb{Z}_{2^{\gamma}} \times \mathbb{Z}_{2^{\min \{\alpha-1, \gamma\}}} \times \mathbb{Z}_{2^{\gamma-1}}$ and $\mathbb{Z}^{6}=\left\langle x_{1}\right\rangle \times \ldots \times\left\langle x_{6}\right\rangle$. Define $\varphi: \mathbb{Z}^{6} \rightarrow L$ by $\varphi=\mu \circ \lambda$, where $\lambda: \mathbb{Z}^{6} \rightarrow \mathbb{Z}^{6}$ is given by $\lambda\left(x_{i}\right)=x_{i}$ for $i=1,2,4,5,6, \lambda\left(x_{3}\right)=x_{3} x_{4}^{-1} x_{5}^{-2} x_{6}^{-2}$, and $\mu: \mathbb{Z}^{6} \rightarrow L$ reduces the generators of $\mathbb{Z}^{6}$ modulo the appropriate powers. Clearly $\lambda$ is an automorphism and $\mu$ is a homomorphism, so $\varphi$ is a homomorphism with $\varphi\left(x_{i}\right)=\epsilon_{i}, i=1, \ldots, 6$, where $\epsilon_{1}=(1,0,0,0,0,0), \ldots, \epsilon_{6}=(0,0,0,0,0,1) \in L$.

Now let $\Gamma=\psi$ be the crossed pairing of Lemma 3.4 with $\psi: \mathcal{H} \times \mathcal{H} \rightarrow \mathbb{Z}^{6}$ and $\pi$ the epimorphism of $\mathcal{H}$ onto $G$. By Proposition 3.1, there exists a crossed pairing $\Delta: G \times G \rightarrow L$ provided

$$
\varphi(\psi(\operatorname{ker} \pi, \mathcal{H}))=\varphi(\psi(\mathcal{H}, \operatorname{ker} \pi))=1
$$

We will now establish (4.2.1). Let $l=\left(l_{1}, \ldots, l_{6}\right) \in L$, and $h, h^{\prime} \in \mathcal{H}$ with $h=x^{m} y^{n} z^{l}$, $h^{\prime}=x^{m^{\prime}} y^{n^{\prime}} z^{l^{\prime}}$, where $m, m^{\prime}, n, n^{\prime}, l, l^{\prime} \in \mathbb{Z}$. By Lemma 3.4 and the definition of $\varphi$ we obtain $\varphi \circ \psi\left(h, h^{\prime}\right)=\varphi\left(x_{1}\left(h, h^{\prime}\right), \ldots, x_{6}\left(h, h^{\prime}\right)\right)=\left(\varphi\left(x_{1}\left(h, h^{\prime}\right)\right), \ldots, \varphi\left(x_{6}\left(h, h^{\prime}\right)\right)\right)=$ $\left(l_{1}\left(h, h^{\prime}\right), \ldots, l_{6}\left(h, h^{\prime}\right)\right)$, where

$$
\begin{aligned}
l_{1}\left(h, h^{\prime}\right) \equiv & m m^{\prime}\left(\bmod 2^{\alpha}\right), \\
l_{2}\left(h, h^{\prime}\right) \equiv & n n^{\prime}\left(\bmod 2^{\gamma}\right), \\
l_{3}\left(h, h^{\prime}\right) \equiv & m n^{\prime}-m^{\prime} n-2\left(n^{\prime}\left(\begin{array}{c}
m \\
2
\end{array}\right)-n\left(\begin{array}{c}
m^{\prime} \\
2
\end{array}\right)+\left(n^{\prime}-n\right) m m^{\prime}+m l^{\prime}-m^{\prime} l\right) \\
& -2\left(m\left(\begin{array}{c}
n^{\prime} \\
2
\end{array}\right)-m^{\prime}\left(\begin{array}{l}
n \\
2
\end{array}\right)+n l^{\prime}-n^{\prime} l\right)\left(\bmod 2^{\gamma+1}\right), \\
l_{4}\left(h, h^{\prime}\right) \equiv & n m^{\prime}\left(\bmod 2^{\gamma}\right), \\
l_{5}\left(h, h^{\prime}\right) \equiv & n^{\prime}\left(\begin{array}{c}
m \\
2
\end{array}\right)-n\left(\begin{array}{c}
m^{\prime} \\
2
\end{array}\right)+\left(n^{\prime}-n\right) m m^{\prime}+m l^{\prime}-m^{\prime} l\left(\bmod 2^{\min \{\alpha-1, \gamma\}}\right), \\
l_{6}\left(h, h^{\prime}\right) \equiv & m\left(\begin{array}{c}
n^{\prime} \\
2
\end{array}\right)-m^{\prime}\left(\begin{array}{l}
n \\
2
\end{array}\right)+n l^{\prime}-n^{\prime} l\left(\bmod 2^{\gamma-1}\right) .
\end{aligned}
$$

Suppose now $h \in \operatorname{ker} \pi$, then $m \equiv 0\left(\bmod 2^{\alpha}\right)$ and $n \equiv l \equiv 0\left(\bmod 2^{\gamma}\right)$. In such a case, since $\alpha \geq \gamma, l_{1}, l_{2}, l_{4}, l_{5}$, and $l_{6}$ are obviously all trivial in $L$. As for $l_{3}$, the terms can be rearranged as

$$
\begin{aligned}
l_{3}\left(h, h^{\prime}\right) & \equiv 2 m\left(m^{\prime} n-m^{\prime} n^{\prime}-l^{\prime}\right)+2 n\left(\left(\begin{array}{c}
m^{\prime}+1 \\
2
\end{array}\right)-l\right)+2 l\left(m^{\prime}+n^{\prime}\right) \\
& -m^{2} n^{\prime}+n n^{2} m^{\prime}\left(\bmod 2^{\gamma+1}\right) .
\end{aligned}
$$

Since $\alpha \geq \gamma$, we have $m \equiv n \equiv l \equiv 0\left(\bmod 2^{\gamma}\right)$. Therefore $2 m \equiv 2 n \equiv 2 l \equiv 0\left(\bmod 2^{\gamma+1}\right)$, and $m^{2} \equiv n^{2} \equiv 0\left(\bmod 2^{\gamma+1}\right)$. Thus $l_{3}$ is trivial in $L$. So $\varphi(\psi(\operatorname{ker} \pi, \mathcal{H}))=1$. In a similar manner we can show $\varphi(\psi(\mathcal{H}, \operatorname{ker} \pi))=1$. Hence (4.2.1) is established.

Since the diagram of Proposition 3.1 commutes for $\Delta$ and $\varphi \circ \psi$ is onto, we conclude that $\Delta$ is onto. Thus by Proposition 1.3, $\Delta$ lifts to a homomorphism $\Delta^{\star}$ of 
$G \otimes G$ onto $L$, and thus $|G \otimes G| \geq|L|$. Since $|G \otimes G| \leq|L|$, it follows that $\Delta^{\star}$ is an isomorphism, and $G \otimes G \cong L$ as claimed.

In the next theorem we determine the tensor square for a group of type (2.5.3). We first prove a lemma establishing order bounds for the generators of the tensor square.

Lemma 4.3. Let $G$ be a group of type (2.5.3). Then $G \otimes G$ is generated by $a \otimes a$, $b \otimes b,(a \otimes b),(a \otimes b)(b \otimes a),(a \otimes a)^{2^{\alpha-\gamma}}(a \otimes[a, b])$ and $(a \otimes b)^{2^{\alpha-\gamma}}(a \otimes[a, b])^{-2^{\alpha-\gamma-1}}$ $(b \otimes[a, b])$. For the orders of the generators the following estimates hold:

$|b \otimes b| \leq 2^{\beta}, \quad\left|(a \otimes a)^{2^{\alpha-\gamma}}(a \otimes[a, b])\right| \leq 2^{\sigma}$,

$\left|(a \otimes b)^{2^{\alpha-\gamma}}(a \otimes[a, b])^{-2^{\alpha-\gamma+1}}(b \otimes[a, b])\right| \leq 2^{\sigma}$, and $|(a \otimes b)(b \otimes a)| \leq 2^{\min \{\alpha-\gamma+\sigma, \beta\}}$.

Furthermore,

$$
\begin{aligned}
& |a \otimes b| \leq \begin{cases}2^{\min \{\alpha, \beta\}}, & \text { if } \alpha \geq \gamma+2 \text { or } \beta \geq \gamma+1, \\
2^{\gamma+1}, & \text { if } \alpha=\gamma+1 \text { and } \beta=\gamma,\end{cases} \\
& |a \otimes a| \leq \begin{cases}2^{\alpha-\gamma+\sigma+1}, & \text { if } \alpha \geq \gamma+2 \text { or } \beta \geq \gamma+1, \\
2^{\gamma}, & \text { if } \alpha=\gamma+1 \text { and } \beta=\gamma .\end{cases}
\end{aligned}
$$

Proof. Our first claim that $G \otimes G$ is generated by the elements in question is an immediate consequence of Lemma 3.3. Next we turn to the estimate for the orders of these generators. If we set $z=[a, b]$, then $c=z a^{-2^{\alpha-\gamma}}$ by (2.5.3). For $b \otimes b$ we have $1_{\otimes}=b^{2^{\beta}} \otimes b=(b \otimes b)^{2^{\beta}}$, the desired estimate.

Next we turn to $(a \otimes a)^{2^{\alpha-\gamma}}(a \otimes[a, b])$. Expanding $c^{2^{\sigma}} \otimes a$ by Lemma 3.2 yields

$$
1_{\otimes}=c^{2^{\sigma}} \otimes a=(a \otimes a)^{-2^{\alpha-\gamma+\sigma}}(z \otimes a)^{2^{\sigma}}=\left((a \otimes a)^{2^{\alpha-\gamma}}(z \otimes a)^{-1}\right)^{-2^{\sigma}} .
$$

However by [2, Lemma 3.3],

$$
([a, b] \otimes a)^{-1}=a \otimes[a, b] .
$$

This together with (4.3.1) gives the desired estimate for this case.

Similarly, expanding $c^{2^{\sigma}} \otimes b$ we obtain

$$
1_{\otimes}=c^{2^{\sigma}} \otimes b=\left((a \otimes b)^{2^{\alpha-\gamma}}(a \otimes z)^{-2^{\alpha-\gamma-1}}(b \otimes z)\right)^{-2^{\sigma}},
$$

providing the desired estimate for the order of $(a \otimes b)^{2^{\alpha-\gamma}}(a \otimes z)^{-2^{\alpha-\gamma-1}}(b \otimes z)$.

We turn now to $(a \otimes b)(b \otimes a)$. Expansion of $b \otimes c^{2^{\sigma}}$ using Lemma 3.2 leads to

$$
1_{\otimes}=b \otimes c^{2^{\sigma}}=(b \otimes a)^{-2^{\alpha-\gamma+\sigma}}(a \otimes z)^{-2^{\alpha-\gamma+\sigma-1}}(b \otimes z)^{2^{\sigma}}
$$

By multiplying (4.3.3) and (4.3.4), we obtain $((a \otimes b)(b \otimes a))^{2^{\alpha-\gamma+\sigma}}=1_{\otimes}$, providing one bound on the order of $(a \otimes b)(b \otimes a)$. To obtain the second bound, we multiply 
the expansions of $1_{\otimes}=a \otimes b^{2^{\beta}}$ and $1_{\otimes}=b^{2^{\beta}} \otimes a$ as given by Lemma 3.2. This yields $1_{\otimes}=((a \otimes b)(b \otimes a))^{2^{\beta}}$.

Next we establish the estimate for the order of $a \otimes b$. Since $\alpha>\gamma$, expansion of $a^{2^{\alpha}} \otimes b$ using Lemma 3.2 yields $1_{\otimes}=\left(a^{2^{\alpha}} \otimes b\right)=(a \otimes b)^{2^{\alpha}}$. This proves our claim if $\alpha=\gamma+1$ and $\beta=\gamma$, and establishes one estimate of the order in the remaining cases, i.e. $\beta \geq \gamma+1$ or $\alpha \geq \gamma+2$. By expanding $a \otimes b^{2^{\beta}}$ using Lemma 3.2, we obtain $1_{\otimes}=(a \otimes b)^{2^{\beta}}(b \otimes z)^{\left(\begin{array}{c}2^{\beta} \\ 2\end{array}\right)}$. If $\beta \geq \gamma+1$, this leads to $1_{\otimes}=(a \otimes b)^{2^{\beta}}$, the desired second bound for the order of $a \otimes b$.

In the remaining case, i.e. $\beta=\gamma$ and $\alpha \geq \gamma+2$, we proceed as follows. Raising (4.3.3) to the power of $2^{\gamma-\sigma-1}$ and inverting yields $1_{\otimes}=(a \otimes b)^{2^{\alpha-1}}(a \otimes z)^{-2^{\alpha-9}}$ $(b \otimes z)^{2^{\gamma-1}}$. Observing $|a \otimes z|<2^{\alpha-1}$ and

$$
1_{\otimes}=\left(a \otimes b^{2^{\gamma}}\right)=(a \otimes b)^{2^{\gamma}}(a \otimes z)^{-2^{\gamma-1}},
$$

together with the above, lead to $1_{\otimes}=(a \otimes b)^{2^{\gamma}\left(2^{\alpha-\gamma+1}+1\right)}$, where $2^{\alpha-\gamma+1}+1$ is odd since $\alpha \geq \gamma+2$. This is the required second bound for $\beta=\gamma$ and $\alpha \geq \gamma+2$.

Finally, we turn to the estimate for the order of $a \otimes a$. Lemma 3.2 gives

$$
1_{\otimes}=a \otimes c^{2^{\sigma}}=(a \otimes a)^{-2^{\alpha-\gamma+\sigma}}(a \otimes z)^{2^{\sigma}}
$$

Equating (4.3.1) and (4.3.6) yields $(a \otimes z)^{2^{\sigma+1}}=1_{\otimes}$. Thus squaring (4.3.1) leads to $(a \otimes a)^{2^{\alpha-\gamma+\sigma+1}}=1_{\otimes}$, which is the desired estimate for $\alpha \geq \gamma+2$ or $\beta \geq \gamma+1$. Now let $\alpha=\gamma+1$ and $\beta=\gamma$. Then $\gamma>\sigma \geq 2 \gamma-\alpha=\gamma-1$, implying $\sigma=\gamma-1$. From (4.3.3) we obtain in this case after inversion $1_{\otimes}=(a \otimes b)^{2^{\gamma}}(a \otimes z)^{2^{\gamma-1}}(b \otimes z)^{2^{\gamma-1}}$. This together with (4.3.5) leads to $1_{\otimes}=(a \otimes b)^{2^{\gamma+1}}(a \otimes z)^{2^{\gamma-1}}$. However, $(a \otimes b)^{2^{\gamma+1}}=1_{\otimes}$ by the above. Therefore $(a \otimes z)^{2^{\gamma-1}}=1_{\otimes}$. Observing (4.3.2), we obtain by (4.3.1) that $1_{\otimes}=(a \otimes a)^{2^{\gamma}}$ in the final case.

TheOREM 4.4. Let $G$ be a group of type (2.5.3), that is $G=(\langle c\rangle \times\langle a\rangle) \rtimes\langle b\rangle$, where $[a, b]=a^{2^{\alpha-\gamma}} c,[c, b]=a^{-2^{2(\alpha-\gamma)}} c^{-2^{\alpha-\gamma}},|a|=2^{\alpha},|b|=2^{\beta},|c|=2^{\sigma},|[a, b]|=2^{\gamma}$, with $\alpha, \beta, \gamma, \sigma \in \mathbb{N}, \alpha+\sigma \geq 2 \gamma, \beta \geq \gamma>\sigma$. Then

$$
G \otimes G \cong \begin{cases}\mathbb{Z}_{2^{\gamma}}^{2} \times \mathbb{Z}_{2^{\gamma+1}} \times \mathbb{Z}_{2^{\gamma}} \times \mathbb{Z}_{2^{\gamma-1}}^{2}, & \text { if } \alpha=\gamma+1 \text { and } \beta=\gamma, \\ \mathbb{Z}_{2^{\alpha-\gamma+\sigma+1}} \times \mathbb{Z}_{2^{\beta}} \times \mathbb{Z}_{2^{\min \{\alpha, \beta\}}} \times \mathbb{Z}_{2^{\rho}} \times \mathbb{Z}_{2^{\sigma}}^{2}, & \text { if } \alpha \geq \gamma+2 \text { or } \beta \geq \gamma+1,\end{cases}
$$

where $\rho=\min \{\alpha-\gamma+\sigma, \beta\}$.

Proof. Let $G$ be a group of type (2.5.3). First notice all cases are covered. If $\alpha<\gamma+2$ then $\alpha=\gamma+1$, since $\gamma+2>\alpha \geq 2 \gamma-\sigma \geq \gamma+1$.

Let $\mathcal{H}=F_{2} / \gamma_{3}\left(F_{2}\right)$. Define $\pi: \mathcal{H} \rightarrow G$ by $\pi(h)=a^{m_{1}} b^{m_{2}} c^{l}$, where $h=x^{m_{1}} y^{m_{2}} v^{l}$ with $m_{1}, m_{2}, l \in \mathbb{Z}, v=x^{-2^{\alpha-\gamma}}[x, y]$, and $a, b, c \in G$ as in the theorem. It follows $\pi$ is an epimorphism with $h \in \operatorname{ker} \pi$ if and only if $m_{1} \equiv 0\left(\bmod 2^{\alpha}\right), m_{2} \equiv 0\left(\bmod 2^{\beta}\right)$, and $l \equiv 0\left(\bmod 2^{\sigma}\right)$. Next, let

$$
L= \begin{cases}\mathbb{Z}_{2^{\gamma}}^{2} \times \mathbb{Z}_{2^{\gamma+1}} \times \mathbb{Z}_{2^{\gamma}} \times \mathbb{Z}_{2^{\gamma-1}}^{2}, & \text { if } \alpha=\gamma+1 \text { and } \beta=\gamma \\ \mathbb{Z}_{2^{\alpha-\gamma+\sigma+1}} \times \mathbb{Z}_{2^{\beta}} \times \mathbb{Z}_{2^{\min \{\alpha, \beta\}}} \times \mathbb{Z}_{2^{\rho}} \times \mathbb{Z}_{2^{\sigma}}^{2}, & \text { if } \alpha \geq \gamma+2 \text { or } \beta \geq \gamma+1\end{cases}
$$


where $\rho=\min \{\alpha-\gamma+\sigma, \beta\}$, and $\mathbb{Z}^{6}=\left\langle x_{1}\right\rangle \times \ldots \times\left\langle x_{6}\right\rangle$. Define $\varphi: \mathbb{Z}^{6} \rightarrow L$ by $\varphi=\mu \circ \lambda$, where $\lambda: \mathbb{Z}^{6} \rightarrow \mathbb{Z}^{6}$ is given by $\lambda\left(x_{1}\right)=x_{1} x_{5}^{-2^{\alpha-\gamma}} \cdot x_{6}^{-2^{2(\alpha-\gamma)-1}}$, $\lambda\left(x_{3}\right)=x_{3} x_{4}^{-1} x_{6}^{-2^{\alpha-\gamma}}, \quad \lambda\left(x_{5}\right)=x_{5} x_{6}^{2^{\alpha-\gamma-1}}, \quad$ and $\lambda\left(x_{i}\right)=x_{i}$ for $i=2,4,6$, while $\mu: \mathbb{Z}^{6} \rightarrow L$ reduces the generators $\lambda\left(x_{i}\right)$ modulo the appropriate 2-powers. Specifically, $\varphi\left(x_{i}\right)=\epsilon_{i}, i=1, \ldots, 6$ where $\epsilon_{1}=(1,0,0,0,0,0), \ldots, \epsilon_{6}=(0,0,0,0,0,1) \in L$. It follows that $\lambda$ is an automorphism of $\mathbb{Z}^{6}$ and $\mu$ is a homomorphism of $\mathbb{Z}^{6}$ onto $L$.

Let $h, h^{\prime} \in \mathcal{H}$ with $h=x^{m_{1}} y^{m_{2}} v^{l}$ and $h^{\prime}=x^{m_{1}^{\prime}} y^{m_{2}^{\prime}} v^{l^{\prime}}$, where as before, $v=x^{-2^{\alpha-\gamma}}[x, y]$. Setting $u=[x, y]$, we obtain alternative presentations for $h$ and $h^{\prime}$ as $h=x^{m} y^{n} u^{k}$ and $h^{\prime}=x^{m^{\prime}} y^{n^{\prime}} u^{k^{\prime}}$, where $m=m_{1}-l 2^{\alpha-\gamma}, m^{\prime}=m_{1}^{\prime}-l^{\prime} 2^{\alpha-\gamma}, n=m_{2}$, $n^{\prime}=m_{2}^{\prime}, \quad k=l$, and $k^{\prime}=l^{\prime}$. By Lemma 3.4, there exists a crossed pairing $\psi: \mathcal{H} \times \mathcal{H} \rightarrow \mathbb{Z}^{6}$, where in terms of the original presentation

$$
\left.\begin{array}{rl}
x_{1}\left(h, h^{\prime}\right)= & \left(m_{1}-l 2^{\alpha-\gamma}\right)\left(m_{1}^{\prime}-l^{\prime} 2^{\alpha-\gamma}\right), \quad x_{2}\left(h, h^{\prime}\right)=m_{2} m_{2}^{\prime}, \\
x_{3}\left(h, h^{\prime}\right)= & \left(m_{1}-l 2^{\alpha-\gamma}\right) m_{2}^{\prime}, \quad x_{4}\left(h, h^{\prime}\right)=\left(m_{1}^{\prime}-l^{\prime} 2^{\alpha-\gamma}\right) m_{2}, \\
x_{5}\left(h, h^{\prime}\right)= & m_{2}^{\prime}\left(\begin{array}{c}
m_{1}-l 2^{\alpha-\gamma} \\
2
\end{array}\right)-m_{2}\left(\begin{array}{c}
m_{1}^{\prime}-l^{\prime} 2^{\alpha-\gamma} \\
2
\end{array}\right)+\left(m_{1}-l 2^{\alpha-\gamma}\right) l^{\prime} \\
& -\left(m_{1}^{\prime}-l^{\prime} 2^{\alpha-\gamma}\right) l+\left(m_{2}^{\prime}-m_{2}\right)\left(m_{1}-l 2^{\alpha-\gamma}\right)\left(m_{1}^{\prime}-l^{\prime} 2^{\alpha-\gamma}\right), \\
x_{6}\left(h, h^{\prime}\right)= & \left(m_{1}-l 2^{\alpha-\gamma}\right)\left(\begin{array}{c}
m_{2}^{\prime} \\
2
\end{array}\right)-\left(m_{1}^{\prime}-l^{\prime} 2^{\alpha-\gamma}\right)\left(\begin{array}{c}
m_{2} \\
2
\end{array}\right)+m_{2} l^{\prime}-m_{2}^{\prime} l .
\end{array}\right\}
$$

We will apply now Proposition 3.1 with $G$ as given in (2.5.3), $H=\mathcal{H}, K=\mathbb{Z}^{6}$, and $L$ as defined earlier in this proof. For the mappings, let $\varphi=\mu \circ \lambda$ and $\Gamma=\psi$, both as given above, and $\pi: \mathcal{H} \rightarrow G$. By Proposition 3.1, there exists a crossed pairing $\Delta: G \times G \rightarrow L$, provided $\varphi(\psi(\operatorname{ker} \pi, \mathcal{H}))=\varphi(\psi(\mathcal{H}, \operatorname{ker} \pi))=1$. We will next show this is the case. Suppose $h, h^{\prime} \in \mathcal{H}$ where $h=x^{m_{1}} y^{m_{2}} v^{l}$ and $h^{\prime}=x^{m_{1}^{\prime}} y^{m_{2}^{\prime}} v^{l^{\prime}}$. Writing $\varphi \circ \psi: \mathcal{H} \times \mathcal{H} \rightarrow L$ componentwise as $\varphi \circ \psi\left(h, h^{\prime}\right)=\left(l_{1}\left(h, h^{\prime}\right), \ldots, l_{6}\left(h, h^{\prime}\right)\right)$, we obtain in terms of $x_{i}\left(h, h^{\prime}\right)$ as given by (4.4.1):

$$
\begin{aligned}
l_{1}\left(h, h^{\prime}\right) \equiv & x_{1}\left(h, h^{\prime}\right)-2^{\alpha-\gamma} x_{5}\left(h, h^{\prime}\right)-2^{2(\alpha-\gamma)-1} x_{6}\left(h, h^{\prime}\right)\left(\bmod 2^{j^{\prime}}\right), \\
& \text { where } j^{\prime}=\gamma, \text { if } \alpha=\gamma+1 \text { and } \beta=\gamma, \text { and } j^{\prime}=\alpha-\gamma+\sigma+1, \text { otherwise; } \\
l_{2}\left(h, h^{\prime}\right) \equiv & x_{2}\left(h, h^{\prime}\right)\left(\bmod 2^{\beta}\right) ; \\
l_{3}\left(h, h^{\prime}\right) \equiv & x_{3}\left(h, h^{\prime}\right)-x_{4}\left(h, h^{\prime}\right)-2^{\alpha-\gamma} x_{6}\left(h, h^{\prime}\right)\left(\bmod 2^{j}\right), \\
& \text { where } j=\gamma+1, \text { if } \alpha=\gamma+1 \text { and } \beta=\gamma, \text { and } j=\min \{\alpha, \beta\}, \text { otherwise; } \\
l_{4}\left(h, h^{\prime}\right) \equiv & x_{4}\left(h, h^{\prime}\right)\left(\bmod 2^{\min \{\alpha-\gamma+\sigma, \beta\}}\right) ; \\
l_{5}\left(h, h^{\prime}\right) \equiv & x_{5}\left(h, h^{\prime}\right)+2^{\alpha-\gamma-1} x_{6}\left(h, h^{\prime}\right)\left(\bmod 2^{\sigma}\right) ; \\
l_{6}\left(h, h^{\prime}\right) \equiv & x_{6}\left(h, h^{\prime}\right)\left(\bmod 2^{\sigma}\right) .
\end{aligned}
$$

Now suppose $h \in \operatorname{ker} \pi$. Then $m_{1} \equiv 0\left(\bmod 2^{\alpha}\right), m_{2} \equiv 0\left(\bmod 2^{\beta}\right)$ and $l \equiv 0\left(\bmod 2^{\sigma}\right)$. It follows directly from the definition of $x_{i}\left(h, h^{\prime}\right)$ that $l_{i}\left(h, h^{\prime}\right)=0$ for $i=2,4,5,6$. We now consider $l_{3}\left(h, h^{\prime}\right)$. Noting that $m_{1}=2^{\alpha} r, m_{2}=2^{\beta} s$ and $l=2^{\sigma} t$ for some $r, s, t \in \mathbb{Z}$, we substitute the explicit values for $x_{3}, x_{4}$ and $x_{6}$ into $l_{3}=x_{3}-x_{4}-2^{\alpha-\gamma} x_{6}$. After simplification we obtain 


$$
\begin{aligned}
l_{3}\left(h, h^{\prime}\right) & =m_{2}^{\prime} 2^{\alpha} r-m_{1}^{\prime} 2^{\beta} s-\left(\begin{array}{c}
m_{2}^{\prime} \\
2
\end{array}\right) 2^{\alpha-\gamma} 2^{\alpha} r+m_{1}^{\prime}\left(\begin{array}{c}
2^{\beta} s \\
2
\end{array}\right) 2^{\alpha-\gamma}+\left(\begin{array}{c}
m_{2}^{\prime} \\
2
\end{array}\right) 2^{\alpha-2 \gamma+\sigma} 2^{\alpha} t \\
& -\left(\begin{array}{c}
2^{\alpha} r \\
2
\end{array}\right) l^{\prime} 2^{2(\alpha-\gamma)} .
\end{aligned}
$$

This leads to

$$
l_{3}\left(h, h^{\prime}\right) \equiv-m_{1}^{\prime} 2^{\beta} s+m_{1}^{\prime}\left(\begin{array}{c}
2^{\beta} s \\
2
\end{array}\right) 2^{\alpha-\gamma}-\left(\begin{array}{c}
2^{\alpha} r \\
2
\end{array}\right) l^{\prime} 2^{2(\alpha-\gamma)}\left(\bmod 2^{j}\right) .
$$

Expanding the binomial coefficients and collecting 2-powers give

$$
l_{3}\left(h, h^{\prime}\right) \equiv m_{1}^{\prime} s^{2} 2^{2 \beta+\alpha-\gamma+1}-m_{1}^{\prime} s\left(1+2^{\alpha-\gamma-1}\right) 2^{\beta}-l^{\prime} r\left(2^{\alpha} r-1\right) 2^{3 \alpha-2 \gamma-1}\left(\bmod 2^{j}\right) .
$$

Since $2 \beta+\alpha-\gamma+1 \geq j$ and $3 \alpha-2 \gamma-1 \geq j$ in both cases, this yields

$$
l_{3}\left(h, h^{\prime}\right) \equiv-m_{1}^{\prime} s\left(1+2^{\alpha-\gamma-1}\right) 2^{\beta}\left(\bmod 2^{j}\right) .
$$

Now if $\alpha>\gamma+1$ or $\beta>\gamma$, clearly $l_{3} \equiv 0\left(\bmod 2^{j}\right)$. However if $\alpha=\gamma+1$ and $\beta=\gamma$, then $l_{3}\left(h, h^{\prime}\right) \equiv-m_{1} s(1+1) 2^{\gamma} \equiv 0\left(\bmod 2^{j}\right)$. In either case $l_{3}\left(h, h^{\prime}\right)=0$. A similar argument shows $l_{1}\left(h, h^{\prime}\right)=0$. Thus $\varphi(\psi(\operatorname{ker} \pi, \mathcal{H}))=1$, as claimed. Similarly, it can be shown that $\varphi(\psi(\mathcal{H}, \operatorname{ker} \pi))=1$. Thus $\Delta$ is a crossed pairing.

Finally, since the diagram of Proposition 3.1 commutes for $\Delta$ and $\psi \circ \varphi$ is onto, we conclude that $\Delta$ is onto. Thus by Proposition $1.3, \Delta$ lifts to a homomorphism $\Delta^{\star}$ of $G \otimes G$ onto $L$, and thus $|G \otimes G| \geq|L|$. However by Lemma 4.3, $|G \otimes G| \leq|L|$. We conclude $\Delta^{\star}$ is an isomorphism, and $G \otimes G \cong L$ as claimed.

It should be mentioned here that the group given in [2, Theorem 4.4] as the tensor square of the $p$-group, $p$ odd, corresponding to a group of type (2.5.3) for $p=2$, is only a homomorphic image of the actual tensor square, namely

$$
G \otimes G \cong \mathbb{Z}_{p^{\alpha-\gamma+\sigma}}^{2} \times \mathbb{Z}_{p^{\beta}} \times \mathbb{Z}_{p^{\min \{\alpha, \beta\}}} \times \mathbb{Z}_{p^{\sigma}}^{2}
$$

This is the same result as in the preceding theorem, where $p=2$ and $\alpha \geq \gamma+2$. The derivation follows in a similar manner.

In our final theorem we compute the tensor squares of groups of type (2.5.4). As these groups are homomorphic images of groups of type (2.5.3), we will obtain their tensor squares as homomorphic images of the tensor squares of the respective groups in (2.5.3).

THEOREM 4.5. Let $G$ be a group of type (2.5.4), that is $G \cong(\langle c\rangle \times\langle a\rangle)\langle b\rangle$, where $|a|=|b|=2^{\gamma+1}, \quad|[a, b]|=2^{\gamma}, \quad|c|=2^{\gamma-1}, \quad[a, b]=a^{2} c, \quad[c, b]=a^{-4} c^{-2}, \quad a^{2^{\gamma}}=b^{2^{\gamma}}$, $\gamma \in \mathbb{N}$. Then

$$
G \otimes G \cong \begin{cases}\mathbb{Z}_{2}^{2} \times \mathbb{Z}_{4}^{2}, & \text { for } \gamma=1, \\ \mathbb{Z}_{2 \gamma}^{3} \times \mathbb{Z}_{2^{\gamma+1}} \times \mathbb{Z}_{2 \gamma-1}^{2}, & \text { for } \gamma \geq 2 .\end{cases}
$$


Proof. Let $\gamma=1$. Then $G$ is the group of quaternions of order 8 and $G \otimes G \cong \mathbb{Z}_{2}^{2} \times \mathbb{Z}_{4}^{2}$ by [3]. Now let $G$ be a group of type (2.5.4) with $\gamma \geq 2$ and $H=(\langle w\rangle \times\langle u\rangle) \rtimes\langle v\rangle$ be a group of type (2.5.3) with $\alpha=\beta=\gamma+1$ and $\sigma=\gamma-1$, that is $|u|=|v|=2^{\gamma+1},|w|=2^{\gamma-1}$ and $|[u, v]|=2^{\gamma}$. Then, by the proof of Theorem 2.5, $\quad \psi: H \rightarrow G$ defined by $\psi\left(w^{r} u^{s} v^{t}\right)=c^{r} a^{s} b^{t}$ is an epimorphism with ker $\psi=\left\langle u^{2 \gamma} v^{2 \gamma}\right\rangle$. It follows from [3, Proposition 1] that there is an epimorphism $\psi \otimes \psi: H \otimes H \rightarrow G \otimes G$. However, ker $\psi$ is central in $H$, since $\left[u^{2^{\gamma}} v^{2^{\gamma}}, h\right]=$ $\left(u^{2^{\gamma}}[v, h]^{2^{\gamma}}\right)[u, h]^{2^{\gamma}}=1$ for all $h \in H$. So, by [3, Proposition 9], $\operatorname{ker}(\psi \otimes \psi) \cong$ $\left\langle u^{2^{\gamma}} v^{2^{\gamma}} \otimes u, u^{2^{\gamma}} v^{2^{\gamma}} \otimes v, u \otimes u^{2^{\gamma}} v^{2^{\gamma}}, v \otimes u^{2^{\gamma}} v^{2^{\gamma}}\right\rangle$.

By Lemma 4.3 and Theorem 4.4 we have $|u \otimes v|=2^{\gamma+1}$ and $\mid(u \otimes v)$. $(v \otimes u) \mid=2^{\gamma}$. Hence

$$
(u \otimes v)^{2^{\gamma}}=(v \otimes u)^{2^{\gamma}}
$$

Expanding the generators of $\operatorname{ker}(\psi \otimes \psi)$, and using (4.5.1) we obtain

$$
u^{2^{\gamma}} v^{2^{\gamma}} \otimes u=(u \otimes u)^{2^{\gamma}}(v \otimes u)^{2^{\gamma}}=(u \otimes u)^{2^{\gamma}}(u \otimes v)^{2^{\gamma}}=u \otimes u^{2^{\gamma}} v^{2^{\gamma}},
$$

and similarly,

$$
u^{2^{\gamma}} v^{2^{\gamma}} \otimes v=(u \otimes v)^{2^{\gamma}}(v \otimes v)^{2^{\gamma}}=(v \otimes u)^{2^{\gamma}}(v \otimes v)^{2^{\gamma}}=v \otimes u^{2^{\gamma}} v^{2^{\gamma}}
$$

Thus $\operatorname{ker}(\psi \otimes \psi)=\left\langle(u \otimes u)^{2^{\gamma}}(u \otimes v)^{2^{\gamma}},(v \otimes v)^{2^{\gamma}}(u \otimes v)^{2^{\gamma}}\right\rangle$, and therefore

$$
G \otimes G \cong H \otimes H /\left\langle(u \otimes u)^{2^{\gamma}}(u \otimes v)^{2^{\gamma}},(v \otimes v)^{2^{\gamma}}(u \otimes v)^{2^{\gamma}}\right\rangle .
$$

Replacing the generators $u \otimes u$ and $v \otimes v$ of $H \otimes H$ by $(u \otimes u)(u \otimes v)$ and $(v \otimes v)(u \otimes v)$, respectively, and observing $H \otimes H \cong \mathbb{Z}_{2^{\gamma+1}}^{3} \times \mathbb{Z}_{2^{\gamma}} \times \mathbb{Z}_{2^{\gamma-1}}^{2}$, it follows by (4.5.2) that $G \otimes G \cong \mathbb{Z}_{2^{\gamma}}^{2} \times \mathbb{Z}_{2^{\gamma+1}} \times \mathbb{Z}_{2^{\gamma}} \times \mathbb{Z}_{2^{\gamma-1}}^{2}$, the desired result.

\section{REFERENCES}

1. R. Aboughazi, Produit tensoriel du group d'Heisenberg, Bull. Soc. Math. France 115 (1987), 95-106.

2. M. Bacon and L.-C. Kappe, The nonabelian tensor square of a 2-generator p-group of class 2, Arch. Math. 61 (1993), 508-516.

3. R. Brown, D. L. Johnson and E. F. Robertson, Some computations of non-abelian tensor products of groups, J. Algebra 111 (1987), 177-202.

4. R. Brown and J.-L. Loday, Excision homotopique en basse dimension, C.R. Acad. Sci. Ser. I Math. Paris 298 (1984), 353-356.

5. R. Brown and J.-L. Loday, Van Kampen theorems for diagrams of spaces, Topology 26 (1987), 311-335.

6. M. Hartl, The nonabelian tensor square and Schur multiplicator of nilpotent groups of class 2, J. Algebra 179 (1996), 416-440.

7. D. L. Johnson, The nonabelian tensor square of a finite split metacyclic group, Proc. Edinburgh Math. Soc. 30 (1987), 91-96.

8. L.-C. Kappe, Nonabelian tensor products of groups: the commutator connection, Groups St. Andrews, 1997 (Vol. 2), London Math. Soc. Lecture Notes 261 (1999), 447-454.

9. D. Y. Trebenko, Nilpotent groups of class two with two generators, Current Analysis and its Applications (Naukova Dumka, Kiev) 228 (1989), 201-208.

10. J. H. C. Whitehead, A certain exact sequence, Ann. Math. 51 (1950), 51-110. 